(i)

Artigo Original

\title{
Avaliação do Impacto do Tratamento com Antivíricos de Ação Direta no Metabolismo da Glicose em Doentes com Infeção Crónica por Vírus da Hepatite C
}

\author{
Joana da Fraga Barreiro a, Dália Fernandes ${ }^{\text {b }}$, Maria Joana Santos ${ }^{\mathrm{c}, *}$ \\ ${ }^{a}$ Escola de Medicina, Universidade do Minho, Braga, Portugal \\ ${ }^{b}$ Serviço de Gastrenterologia, Hospital de Braga, Braga, Portugal \\ 'Assistente Hospitalar, Serviço de Endocrinologia, Hospital de Braga, Braga, Portugal
}

\section{INFORMAÇÃO SOBRE O ARTIGO}

\section{Historial do artigo:}

Received/ Recebido: 2021-03-29

Accepted/Aceite: 2021-12-13

Publicado / Published: 2022-01-24

C Autor (es) (ou seu (s) empregador (es)) e Revista SPEDM 2021. Reutilização permitida de acordo com CC BY-NC. Nenhuma reutilização comercial

(C) Author(s) (or their employer(s)) and SPEDM Journal 2021. Re-use permitted under CC BY-NC.

No commercial re-use.

Palavras-chave:

Antivirais;

Diabetes Mellitus;

Efeitos Colaterais e Reações Adversas Relacionados a

Medicamentos;

Hepatite C Crónica/tratamento farmacológico.

Keywords:

Antiviral Agents;

Drug-Related Side Effects and Adverse Reactions;

Hepatitis C, Chronic/drug therapy.

\section{R E S U M O}

Introdução: A literatura reporta uma associação entre a infeção crónica por vírus da hepatite $\mathrm{C}$ (VHC) e diabetes mellitus tipo 2 (DM2) ou insulinorresistência. Curar a infeção crónica por VHC com interferão associa-se a melhoria de várias alterações hepáticas e metabólicas. Recentemente, o tratamento da infeção por VHC foi revolucionado pelo desenvolvimento de agentes antivíricos de ação direta (AAD), que possuem elevada probabilidade de eliminação sustentada do vírus. O principal objetivo deste trabalho é analisar o impacto do tratamento com AAD no metabolismo da glicose em doentes com infeção crónica por VHC e DM2/pré-diabetes.

Material e Métodos: Incluímos 138 doentes que realizaram erradicação vírica com AAD entre $1 / 1 / 2015$ e 31/8/2019. Recolhemos variáveis para caracterização da amostra, relativas à infeção por VHC, status hepático e status metabólico.

Resultados: Cinquenta quatro doentes $(39,1 \%)$ tinham alterações do metabolismo da glicose. Após a eliminação vírica, verificou-se uma melhoria significativa da fibrose hepática $(p=0,001)$, diminuição significativa da ALT $(p<0,001)$, AST $(p<0,001)$ e GGT $(p=0,001)$ e diminuição significativa da glicemia em jejum $(p<0,001)$. A fibrose inicial correlacionou-se significativamente e de forma negativa com a variação da glicemia em jejum após o tratamento $(p=0,032)$. Não foi possível encontrar preditores de melhoria do controlo glicémico.

Conclusão: Neste estudo, verificámos melhoria do controlo glicémico após a cura da infeção por VHC, assim como normalização dos parâmetros hepáticos analíticos e regressão da fibrose hepática. Estudos prospetivos devem ser realizados para averiguar a sustentabilidade desta resposta e identificar possíveis preditores da melhoria do controlo metabólico destes doentes.

\section{Impact of Direct-Acting Antiviral Treatment on Glucose Metabolism in Patients with Chronic Hepatitis C Virus Infection}

A B S T R A C T

Introduction: An association between hepatitis $\mathrm{C}$ virus (HCV) infection and type 2 diabetes mellitus (DM2) or insulin resistance has been reported. Curing HCV chronic infection with interferon is associated with an improvement in various hepatic and metabolic parameters. The treatment of $\mathrm{HCV}$ infection has recently been revolutionized by the development of direct-acting antiviral agents (AADs), which have a high probability of sustained HCV elimination. The main aim of this study is to analyze the impact of DAA treatment on glucose metabolism in patients with chronic HCV infection and DM2/pre-diabetes.

\footnotetext{
*Autor Correspondente / Corresponding Author. E-Mail: mjoanasantos@hotmail.com (Maria Joana Santos) Sete Fontes - São Victor 4710-243 Braga, Portugal
} 
Material and methods: This study included 138 patients who underwent virus eradication with DAA between $1 / 1 / 2015$ and 31/8/2019. We evaluated data for sample characterization, including HCV infection, liver and metabolic status related data.

Results: Fifty four patients (39.1\%) had impaired glucose metabolism. Following viral clearance, there was a significant improvement in liver fibrosis $(p=0.001)$, significant reduction in ALT $(p<0.001)$, AST $(p<0.001)$ and GGT $(p 0.001)$ and fasting glucose $(p<0.001)$. Initial fibrosis was negatively correlated with fasting glucose variation after treatment $(p=0.032)$. We could not find any predictors of improved glycemic control.

Conclusion: In this study, there was an improvement in glycemic control after the cure of HCV infection, as well as normalization of analytical liver parameters and regression of hepatic fibrosis. Prospective studies should be performed to assess the sustainability of these improvements and identify possible predictors of improved metabolic control.

\section{Introdução \\ Hepatite C}

A hepatite $\mathrm{C}$ é uma doença causada pelo vírus da hepatite $\mathrm{C}$ (VHC), que pode provocar hepatite aguda ou crónica. Cerca de $70 \%$ dos indivíduos infetados desenvolverão hepatite $\mathrm{C}$ crónica. ${ }^{1}$ O objetivo primário do tratamento antivírico é curar a infeção, com a obtenção de resposta virológica sustentada (RVS), definida como ácido ribonucleico (RNA) do VHC indetetável às 12 ou 24 semanas após o fim do tratamento. A erradicação da infeção por VHC previne as suas complicações hepáticas e extra-hepáticas, melhora a qualidade de vida do doente e evita a sua transmissão. O tratamento desta infeção foi recentemente revolucionado pelo desenvolvimento de agentes antivíricos de ação direta (AAD), que possuem elevada probabilidade de eliminação sustentada do VHC. ${ }^{2}$ Os esquemas terapêuticos anteriores, com interferão alfa peguilado (INF) e ribavirina, eram mal tolerados, tinham vários efeitos adversos e taxas de cura entre 40\%-65\%. ${ }^{3}$ Atualmente, todos os doentes com infeção por VHC devem ser considerados para tratamento com $\mathrm{AAD}$, incluindo doentes que não responderam a tratamentos anteriores. A escolha do esquema terapêutico depende do genótipo do $\mathrm{VHC}$, gravidade da doença hepática e existência de tratamento anterior. ${ }^{4}$

\section{Diabetes, Pré-diabetes e Resistência à Insulina}

A diabetes mellitus (DM) representa um grupo de doenças metabólicas que se caracterizam por hiperglicemia resultante de defeitos na secreção e/ou na ação da insulina. ${ }^{5}$ Diagnostica-se com valores de glicemia em jejum iguais ou superiores a $126 \mathrm{mg} / \mathrm{dL}$, valores de glicose igual ou superior a $200 \mathrm{mg} / \mathrm{dL}$ numa prova de tolerância à glicose oral (PTGO) 2 horas após a ingestão de $75 \mathrm{~g}$ de glicose, hemoglobina glicosilada (HbA1c) igual ou superior a $6,5 \%$ ou pela existência de sintomas clássicos de hiperglicemia com glicose plasmática igual ou superior a $200 \mathrm{mg} / \mathrm{dL}$.

"Pré-diabetes" e "Hiperglicemia intermédia" são termos usados para classificar indivíduos que apresentam anomalia da glicemia em jejum (AGJ) (glicemia em jejum entre 100 e $125 \mathrm{mg} / \mathrm{dL}$ ), tolerância diminuída à glicose (TDG) (glicemia entre 140 e 199 $\mathrm{mg} / \mathrm{dL}$ numa PTGO 2 horas após a ingestão de $75 \mathrm{~g}$ de glicose) e/ ou $\mathrm{HbA} 1 \mathrm{c}$ entre $5,7 \%-6,4 \%{ }^{6}$

Insulinorresistência (IR) define-se como uma resposta subnormal da glicose a concentrações normais de insulina endógena e/ou exógena. Está frequentemente associada a obesidade, mas pode resultar de várias outras causas. ${ }^{7}$ Representa um importante problema de saúde pública, uma vez que desempenha um papel central na fisiopatologia da DM2 e está ainda associada a aumento do risco cardiovascular, dislipidemia e síndrome metabólico. ${ }^{8}$

\section{DM e VHC}

Vários estudos mostraram existir uma associação significativa entre a infeção por VHC e a DM ou IR. ${ }^{9}$ A prevalência de DM2 em doentes com $\mathrm{VHC}$ positivo varia entre $2.9 \%-12.6 \%$ e cerca de $30 \%$ dos infetados com VHC têm anomalias do metabolismo da glicose (DM ou AGJ). ${ }^{10} \mathrm{~A}$ infeção crónica por VHC associa-se a um aumento da resistência periférica e hepática à insulina. ${ }^{11} \mathrm{O}$ VHC pode promover IR por vários mecanismos: inibição direta da via de sinalização da insulina no hepatócito; produção excessiva de citocinas pró-inflamatórias; stress oxidativo; modulação de incretinas e disfunção de células beta do pâncreas. ${ }^{10,12,13}$ A presença de IR em doentes infetados com o VHC está associada a pior prognóstico, progressão da fibrose hepática, diminuição da resposta ao tratamento e maior risco de carcinoma hepatocelular. ${ }^{9,10,13}$

A fibrose hepática severa e a cirrose podem, por si só, comprometer o metabolismo da glicose, mas não serão os únicos fatores a explicar a relação VHC/hiperglicemia, uma vez que na cirrose por outras causas, o risco de alterações do metabolismo da glicose é menor. ${ }^{9}$ Estudos sugerem que a eliminação do VHC em doentes com hepatite $\mathrm{C}$ crónica tratados com INF se associa a melhoria de várias alterações metabólicas, como redução de $2 / 3$ do risco de incidência de DM2, independentemente da idade, presença de cirrose e pré-diabetes anterior ao tratamento. ${ }^{14}$ Por outro lado, em doentes sem resposta ao tratamento antivírico, verificou-se uma maior incidência de DM e IR.$^{13}$ Contudo, permanece por esclarecer se o tratamento com fármacos antivíricos de ação direta tem o mesmo efeito a longo prazo na evolução da DM2. ${ }^{15}$

Este trabalho teve como objetivo analisar o impacto do tratamento com antivíricos de ação direta no metabolismo da glicose em doentes com infeção crónica por VHC e pré-diabetes/DM2, nomeadamente analisar a evolução longitudinal dos parâmetros hepáticos e metabólicos após o tratamento nos doentes com pré-diabetes/DM2 e determinar fatores preditivos de melhoria do metabolismo da glicose.

\section{Material e Métodos}

Realizámos um estudo observacional e retrospetivo, que incluiu os doentes com infeção crónica por VHC que realizaram erradicação com AAD entre 1/1/2015 e 31/8/2019, seguidos na consulta de Gastrenterologia do Hospital de Braga. Excluímos os doentes com impossibilidade de consulta do processo clínico ou perda de seguimento; ausência de registo que permitisse conclusões acerca do metabolismo da glicose anteriormente ao tratamento; coinfeção VHB; doença endócrina que potencialmente afetasse o metabolismo da glicose ou induzisse insulinorresistência; doentes sob corticoterapia; grávidas e doentes com DM que não tipo 2 ou cujo esquema terapêutico atual contemplasse interferão. 
Apenas considerámos válidos valores de hemoglobina glicosilada em doentes sem hemoglobinopatias ou outras situações com elevado turnover eritrocitário que potencialmente afetassem o valor deste parâmetro.

Foram recolhidos, com recurso ao processo eletrónico dos doentes, dados relativos a:

- Demografia/dados antropométricos: sexo; idade à data do tratamento; Índice de Massa Corporal (IMC) inicial; peso antes e após tratamento com AAD.

- Infeção por VHC: tempo após diagnóstico de infeção até realização de tratamento; genótipo do VHC; tratamentos prévios; esquema terapêutico atual com AAD; carga vírica antes e após tratamento.

- Citólise e função hepática (antes e após fim de tratamento antivírico): Aspartato aminotransferase (AST); Alanina aminotransferase (ALT); Bilirrubina total (BT); Albumina; rácio normalizado internacional (INR); Fosfatase alcalina (FA); Gama glutamil transferase (GGT). Dada a natureza retrospetiva do estudo, consideraram-se valores até 15 meses antes do tratamento e 3-15 meses após conclusão do mesmo.

- Fibrose hepática: avaliada por elastografia hepática transitória $\left(\right.$ Fibroscan $^{\circ}$ ) antes e após fim do tratamento; consideraram-se os valores mais próximos do início e fim do tratamento.

- Metabolismo da glicose prévio: diagnóstico prévio de DM2 ou pré-DM2 ou critérios diagnósticos com base em dados existentes no processo clínico

- Tratamento para DM2 (antes e após fim de tratamento antivírico):

- Controlo glicémico (antes e após fim de tratamento antivírico): glicose em jejum e HbAlc. Consideraram-se valores antes do tratamento até um período máximo de 15 meses e entre 3-15 meses após término do mesmo. Foram excluídas análises em contexto de urgência/ internamento hospitalar. Nos doentes diabéticos com valores de HbA1c e cujo esquema terapêutico antivírico incluía ribavirina foi avaliada a presença de anemia hemolítica de forma a assegurar a veracidade desse valor.

Nas variáveis quantitativas contínuas avaliámos a existência de distribuição normal através do histograma e teste de Shapiro-Wilk. As variáveis contínuas com distribuição não normal são apresentadas com mediana, percentil 25, percentil 75 e valores mínimo e máximo. As variáveis contínuas com distribuição normal são apresentadas com média, desvio padrão e valores mínimo e máximo. Para variáveis categóricas, apresentam-se número absoluto e percentagem. Para comparar variáveis contínuas e ordinais no mesmo grupo em dois tempos diferentes utilizámos o teste de Wilcoxon. Para comparar variáveis contínuas e ordinais entre dois grupos independentes utilizámos o teste de Mann-Whitney e teste $t$ de Student. Para comparar variáveis nominais entre dois grupos utilizámos o teste do qui quadrado e o teste exato de Fisher. Recorremos à correlação de Spearman para averiguar relação entre 2 variáveis contínuas de distribuição não normal e 1 variável contínua e 1 variável ordinal. Esta relação foi considerada proporcional quando o sinal é positivo e inversa quando negativo, baixa quando $r$ inferior a 0,3 , moderada quando $r$ entre 0,3 e 0,7 e elevada quando $r$ superior a 0,7 . Por último, procedemos à construção de modelos de regressão logística para determinar fatores preditores de uma redução $\geq 7 \%$ na glicemia em jejum no período de follow-up pós-tratamento. Realizámos a análise estatística através do software IBM SPSS ${ }^{\circledR}$ versão 26.0. Considerámos um intervalo de confiança de $95 \%$, com significância estatística para valores de $p<0,05$.

\section{Resultados}

Analisámos 330 processos de doentes propostos para tratamento com antivíricos de ação direta entre 1/1/2015 e 31/8/2019. Destes, 157 não possuíam análises anteriores ao tratamento que permitissem conclusões acerca do metabolismo da glicose; 6 não se inseriram no período de follow-up; 7 realizaram esquemas que contemplavam interferão; 1 tinha coinfecção com VHB; 17 não realizaram ou não concluíram tratamento; 1 tinha diabetes tipo 1; 1 não tinha informação no processo clínico e 2 tiveram perda de seguimento. Analisámos os processos dos 138 restantes doentes, sendo que $54(39,1 \%)$ apresentavam alterações do metabolismo da glicose (pré-DM ou DM2), pelo que foram incluídos neste estudo. Trinta e um $(22,5 \%)$ tinham critérios de pré-DM2 e 16,6\% tinham DM2. Todos os doentes com pré-DM2 apresentavam alteração da glicose em jejum. No grupo de doentes com DM2, 21 estavam medicados, a maioria com apenas 1 fármaco, sendo a metformina $(52,4 \%)$ e os iDPP4 $(52,4 \%)$ as classes farmacológicas mais usadas.

A Tabela 1 mostra as características demográficas, relacionadas com a infeção por VHC, status hepático e metabólico prévios ao tratamento dos 138 doentes analisados e, mais concretamente, dos 54 doentes com alterações do metabolismo da glicose. O genótipo mais comum foi o genótipo $1 \mathrm{a}(39,1 \%)$, constatando-se que 102 doentes $(73,9 \%)$ possuíam genótipo 1 ou um dos seus subtipos $(1,1 \mathrm{a}$ ou 1b). Por sua vez, na escolha do esquema terapêutico verificámos que a grande maioria realizou LED+SOF (63\%), sendo que 117 doentes $(84,6 \%)$ ledipasvir + sofosbuvir (LED + SOF) realizaram esquemas que incluíram SOF. Setenta e um por cento dos doentes nunca tinha realizado tratamento prévio.

Quando comparado o grupo com alterações do metabolismo da glicose com o grupo sem estas alterações, verificámos que no primeiro grupo havia uma percentagem significativamente maior de homens $(\mathrm{p}=0,015)$, doentes mais velhos $(56,4$ versus $52,4, p=0,031)$ e cirrose hepática $(41,7 \%$ versus $24,7 \%, p=0,002)$ (Tabela 1$)$.

No grupo com DM2 também se verificou uma maior percentagem de homens, valores mais elevados de glicemia em jejum, colestase mais acentuada e marcadores de função hepática mais deteriorados (GGT $p=0,024, \mathrm{FA} p=0,39)$, com valores de INR aumentados $(p=0,014)$ e de albumina diminuídos $(p<0,001)$ e maior prevalência de cirrose $(21,4 \%$ versus $70 \%, p<0,001)$. (Tabela 1 )

\section{Evolução longitudinal dos parâmetros hepáticos e metabólicos após o tratamento em doentes com alteração do metabolismo da glicose}

Trinta doentes com AGJ $(96,8 \%)$ e 21 doentes (91,3\%) com DM2 tiveram RVS $(p=0,569)$. Os restantes 3 doentes não tiveram RVS, pelo que foram excluídos da análise posterior. Verificámos uma diminuição significativa da carga vírica após conclusão do tratamento $(p<0,001)$. A Tabela 2 mostra a evolução status hepático antes e após tratamento nos doentes com alteração do metabolismo da glicose. Os parâmetros ALT, AST e GGT melhoraram significativamente após a realização do tratamento, quer nos doentes com alteração da glicose em jejum, quer nos doentes com DM2 (Fig. 1). Também a fibrose hepática diminuiu significativamente após conclusão do tratamento, em todos os subgrupos analisados, verificando-se que globalmente aumentou o número de indivíduos em estadio F0/F1 e diminuiu o número de indivíduos em estadio F4 (Fig.2). No que concerne aos valores de FA, BT, albumina e INR não verificámos alterações significativas após conclusão do tratamento, excetuando a FA 
Tabela 1. Características demográficas, relativas à infeção por VHC, status hepático e status metabólico pré-tratamento dos doentes sujeitos a tratamento antivírico.

\begin{tabular}{|c|c|c|c|c|c|c|}
\hline \multirow{2}{*}{ Características demográficas } & \multicolumn{3}{|c|}{ Alterações do metabolismo da glicose } & \multicolumn{3}{|c|}{$\begin{array}{c}\text { Doentes com alterações do } \\
\text { metabolismo da glicose }(n=54)\end{array}$} \\
\hline & $\begin{array}{c}\text { Não } \\
(n=84)\end{array}$ & $\begin{array}{c}\operatorname{Sim} \\
(\mathrm{n}=54)\end{array}$ & $p$ & $\begin{array}{c}\text { AGJ } \\
(\mathrm{n}=31)\end{array}$ & $\begin{array}{c}\text { DM2 } \\
(\mathrm{n}=\mathbf{2 3})\end{array}$ & $p$ \\
\hline \multicolumn{7}{|l|}{ Sexo } \\
\hline Masculino & $54(64,3 \%)$ & $45(83,3 \%)$ & 0,015 & $23(74,2 \%)$ & $22(95,7 \%)$ & NS \\
\hline Feminino & $30(35,7 \%)$ & $9(16,7 \%)$ & & $8(25,8 \%)$ & $1(4,3 \%)$ & \\
\hline Idade (anos)* & $\begin{array}{c}52,4 \pm 11.9 \\
(26-85)\end{array}$ & $\begin{array}{c}56,4 \pm 10.1 \\
(32-82)\end{array}$ & 0,037 & $\begin{array}{l}55 \pm 10 \\
(32-78) \\
\end{array}$ & $\begin{array}{c}58,1 \pm 10,3 \\
(43-82)\end{array}$ & NS \\
\hline \multicolumn{7}{|l|}{ Infeção VHC } \\
\hline Tempo desde diagnóstico até tratamento (anos)** & $\begin{array}{c}\mathrm{n}=59 \\
17(5-21)(1-29) \\
\end{array}$ & $\begin{array}{c}\mathrm{n}=41 \\
8(3-21,5)(1-32) \\
\end{array}$ & NS & $\begin{array}{c}\mathrm{n}=24 \\
7.5(3,3-21)(1-32)\end{array}$ & $\begin{array}{c}\mathrm{n}=17 \\
16(1,5-25)(1-31) \\
\end{array}$ & NS \\
\hline Carga vírica (UI)** & $\begin{array}{c}\mathrm{n}=82 \\
1860630(392912,8- \\
4740446,3) \\
(482-32300682)\end{array}$ & $\begin{array}{c}\mathrm{n}=53 \\
2725988(509260- \\
4437748) \\
(128-24374591)\end{array}$ & NS & $\begin{array}{c}\mathrm{n}=31 \\
3154937(877436- \\
4909393)(19133- \\
24374591)\end{array}$ & $\begin{array}{c}\mathrm{n}=22 \\
2233939,5 \\
(927056,75- \\
3544540) \\
(128-9270580) \\
\end{array}$ & NS \\
\hline \multicolumn{7}{|l|}{ Status hepático pré-tratamento } \\
\hline $\operatorname{AST}(\mathrm{UI} / \mathrm{L}) * *$ & $\begin{array}{c}\mathrm{n}=79 \\
53(28-80) \\
(13-340)\end{array}$ & $\begin{array}{c}\mathrm{n}=52 \\
61.5(37,8-82,3) \\
(16,0-331,0)\end{array}$ & NS & $\begin{array}{c}\mathrm{n}=29 \\
50(37-83,5) \\
(19-331)\end{array}$ & $\begin{array}{c}\mathrm{n}=23 \\
64(43-84) \\
(16-138)\end{array}$ & NS \\
\hline $\operatorname{ALT}(\mathrm{UI} / \mathrm{L}) * *$ & $\begin{array}{c}\mathrm{n}=78 \\
87(50-140) \\
(16-458)\end{array}$ & $\begin{array}{c}\mathrm{n}=52 \\
99,5(55-138.8) \\
(26,0-388,0)\end{array}$ & NS & $\begin{array}{c}\mathrm{n}=29 \\
98(56-141,5) \\
(26-388)\end{array}$ & $\begin{array}{c}\mathrm{n}=23 \\
112(54-138) \\
(27-256)\end{array}$ & NS \\
\hline GGT (UI/L)** & $\begin{array}{c}n=67 \\
85(36-131) \\
(14-1216)\end{array}$ & $\begin{array}{c}\mathrm{n}=46 \\
114,5(71,0-196,0) \\
(20,0-1027,0)\end{array}$ & 0,011 & $\begin{array}{c}\mathrm{n}=27 \\
103(51-120) \\
(20-539)\end{array}$ & $\begin{array}{c}n=19 \\
167(84-252) \\
(36-1027)\end{array}$ & 0,024 \\
\hline $\mathrm{FA}(\mathrm{UI} / \mathrm{L})^{* *}$ & $\begin{array}{c}\mathrm{n}=67 \\
83(69-102) \\
(34-212)\end{array}$ & $\begin{array}{c}\mathrm{n}=46 \\
75.5(64,8-95,3) \\
(38,0-274,0)\end{array}$ & NS & $\begin{array}{c}\mathrm{n}=26 \\
69.5(62,5-85,3) \\
(53-139)\end{array}$ & $\begin{array}{c}\mathrm{n}=20 \\
87(70,3-123) \\
(38-274)\end{array}$ & 0,039 \\
\hline $\mathrm{BT}(\mathrm{mg} / \mathrm{dL})^{* *}$ & $\begin{array}{c}\mathrm{n}=72 \\
0.6(0.5-0.8) \\
(0.2-3,1) \\
\end{array}$ & $\begin{array}{c}\mathrm{n}=49 \\
0.7(0,5-0,8) \\
(0,4-1,5)\end{array}$ & NS & $\begin{array}{c}\mathrm{n}=28 \\
0,7(0,5-0,8) \\
(0,4-1,1)\end{array}$ & $\begin{array}{c}\mathrm{n}=21 \\
0,7(0,5-0,9) \\
(0,4-1,5)\end{array}$ & NS \\
\hline Albumina $(\mathrm{mg} / \mathrm{dL})^{* *}$ & $\begin{array}{c}\mathrm{n}=63 \\
4.0(3.7-4.1) \\
(1.2-4.7) \\
\end{array}$ & $\begin{array}{c}(\mathrm{n}=44) \\
3.9(3.7-4.2) \\
(3.3-4.7) \\
\end{array}$ & NS & $\begin{array}{c}\mathrm{n}=25 \\
4.1(3.9-4.3) \\
(3.3-4.7) \\
\end{array}$ & $\begin{array}{c}\mathrm{n}=19 \\
3,7(3,6-3,9) \\
(3,3-4,3) \\
\end{array}$ & $<0,001$ \\
\hline $\mathrm{INR}^{* *}$ & $\begin{array}{c}\mathrm{n}=63 \\
1,10(1,00-1,20) \\
(0,85-3,10) \\
\end{array}$ & $\begin{array}{c}\mathrm{n}=39 \\
1,0(1,0-1,1) \\
(0,9-1,2)\end{array}$ & 0.014 & $\begin{array}{c}\mathrm{n}=23 \\
1.00(1,00-1,03) \\
(0,90-1,100)\end{array}$ & $\begin{array}{c}\mathrm{n}=16 \\
1,07(1,00-1,10) \\
(0,90-1,20) \\
\end{array}$ & 0,014 \\
\hline $\begin{array}{l}\text { Fibrose hepática } \\
\text { F0/F1 } \\
\text { F1 } \\
\text { F1/F2 } \\
\text { F2 } \\
\text { F2/F3 } \\
\text { F3 } \\
\text { F3/F4 } \\
\text { F4 } \\
\end{array}$ & $\begin{array}{c}\mathrm{n}=73 \\
33(45,2 \%) \\
4(5,5 \%) \\
7(9,6 \%) \\
3(4,1 \%) \\
1(1,4 \%) \\
4(5,5 \%) \\
3(4,1 \%) \\
18(24,7 \%) \\
\end{array}$ & $\begin{array}{c}\mathrm{n}=48 \\
10(20,8 \%) \\
- \\
2(4,2 \%) \\
3(6,3 \%) \\
1(2,1 \%) \\
11(22,9 \%) \\
1(2,1 \%) \\
20(41,7 \%) \\
\end{array}$ & 0.002 & $\begin{array}{c}\mathrm{n}=28 \\
9(32,1 \%) \\
- \\
2(7,1 \%) \\
3(10,7 \%) \\
- \\
7(25 \%) \\
1(3,6 \%) \\
6(21,4 \%) \\
\end{array}$ & $\begin{array}{c}\mathrm{n}=20 \\
1(5 \%) \\
- \\
- \\
- \\
1(5 \%) \\
4(20 \%) \\
- \\
14(70 \%) \\
\end{array}$ & $<0,001$ \\
\hline \multicolumn{7}{|l|}{ Status metabólico pré-tratamento } \\
\hline Índice de massa corporal $\left(\mathrm{kg} / \mathrm{m}^{2}\right)^{* *}$ & $\begin{array}{c}\mathrm{n}=61 \\
24,8(22,0-28,8) \\
(18,6-36,5)\end{array}$ & $\begin{array}{c}\mathrm{n}=39 \\
25,1(22,8-29,0) \\
(16,5-45,4)\end{array}$ & NS & $\begin{array}{c}\mathrm{n}=21 \\
27,6(23,5-29,5) \\
(16,5-35,3)\end{array}$ & $\begin{array}{c}\mathrm{n}=18 \\
24,31(22,0-27,0) \\
(16,7-45,4)\end{array}$ & NS \\
\hline Indice de massa corporal $\left(\mathrm{kg} / \mathrm{m}^{2}\right)$ em categorias & $\mathrm{n}=61$ & $\mathrm{n}=39$ & & $\mathrm{n}=21$ & $\mathrm{n}=18$ & \\
\hline Baixo peso & - & $2(5,1 \%)$ & & $1(4,8 \%)$ & $1(5,6 \%)$ & \\
\hline Peso normal & $32(52,5 \%)$ & $17(43,6 \%)$ & & $8(38,1 \%)$ & $9(50 \%)$ & \\
\hline Excesso de peso & $18(29,5 \%)$ & $13(33,3 \%)$ & NS & $8(31,8 \%)$ & $5(27,8 \%)$ & NS \\
\hline Obesidade grau I & $10(16,4 \%)$ & $5(12,8 \%)$ & & $3(14,3 \%)$ & $2(11,1 \%)$ & \\
\hline Obesidade grau II & $1(1,6 \%)$ & $1(2,6 \%)$ & & $1(4,8 \%)$ & - & \\
\hline Obesidade grau III & - & $1(2,6 \%)$ & & - & $1(5,6 \%)$ & \\
\hline Peso $(\mathrm{kg})^{* *}$ & $\begin{array}{c}\mathrm{n}=62 \\
67,4(61,8-82,5) \\
(50-108,5)\end{array}$ & $\begin{array}{c}\mathrm{n}=39 \\
70(64-85)(45-128)\end{array}$ & NS & $\begin{array}{c}71,4(65,5-92) \\
(45-114)\end{array}$ & $\begin{array}{c}67,5(60-73,9) \\
(50-128)\end{array}$ & NS \\
\hline Glicemia em jejum $(\mathrm{mg} / \mathrm{dL})^{* *}$ & $\begin{array}{c}\mathrm{n}=83 \\
87(80-92) \\
(62-99) \\
\end{array}$ & $\begin{array}{c}\mathrm{n}=50 \\
108(103-131,5) \\
(74-257)\end{array}$ & $<0,001$ & $\begin{array}{c}\mathrm{n}=31 \\
105(103-111) \\
(100-126)\end{array}$ & $\begin{array}{c}\mathrm{n}=19 \\
141(121-189) \\
(74-257) \\
\end{array}$ & $<0,001$ \\
\hline $\mathrm{HbAlc}(\%)^{* *}$ & $\begin{array}{c}\mathrm{n}=3 \\
5,2(4,9-)(4,9-5,2)\end{array}$ & $\begin{array}{c}\mathrm{n}=19 \\
6,5(5,8-7,3)(5.4-9.2)\end{array}$ & 0,006 & & $\begin{array}{c}\mathrm{n}=19 \\
141(121-189) \\
(74-257) \\
\end{array}$ & \\
\hline
\end{tabular}

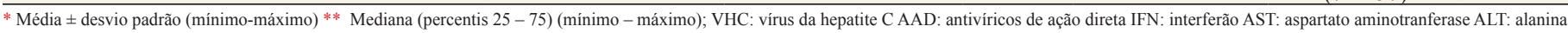
aminotranferase GGT: gama glutamil transferase FA: fosfatase alcalina BT: bilirrubina total INR: razão normalizada internacional HbA1c: hemoglobina glicosilada NS: não significativo 
Tabela 2. Comparação do status metabólico antes e após tratamento.

\begin{tabular}{|c|c|c|c|}
\hline Status metabólico & Antes & Após & $p$ \\
\hline \multicolumn{4}{|c|}{ Glicemia em jejum (mg/dL)** } \\
\hline Todos $(\mathrm{N}=27)$ & $111(104-126)(101-197)$ & $101(91-115)(68-178)$ & $<0,001$ \\
\hline Pré DM2 (n=17) & $108,0(103,5-113,5)(102,0-121,0)$ & $100,0(92.0-106.5)(68.0-117.0)$ & 0,002 \\
\hline DM2 $(n=10)$ & $132(117,0-177,8)(101,0-197,0)$ & $114.5(90.5-128.5)(77.0-178.0)$ & 0,007 \\
\hline \multicolumn{4}{|l|}{ HbA1c $(\%)^{* *}$} \\
\hline DM2 $(n=11)$ & $6,1(5,8-6,9)(5,5-7,7)$ & $5,9(5,7-6,2)(5,2-6,7)$ & NS \\
\hline \multicolumn{4}{|l|}{ Peso $(\mathbf{k g}) * *$} \\
\hline Todos $(n=26)$ & $72,9(66,8-93,5)(58,0-128,0)$ & $74,9(67,1-91,3)(57,0-115,0)$ & NS \\
\hline $\operatorname{AGJ}(n=16)$ & $74,0(67,3-96,5)(58,3-114,5)$ & $74,9(68,5-93,0)(62,0-115,0)$ & NS \\
\hline $\mathrm{DM} 2(\mathrm{n}=10)$ & $72,8(61,5-88,5)(58,0-128,0)$ & $75,4(64,0-91,3)(57,0-108,0)$ & NS \\
\hline
\end{tabular}

no subgrupo de doentes diabéticos que se mostrou significativamente menor $(p=0,011)$.

Seguidamente, procedemos à comparação dos parâmetros metabólicos antes e após o tratamento com AAD (Tabela 2). Foram excluídos desta comparação 6 doentes: 2 não tinham informação acerca da medicação no período pós-tratamento; 2 não tiveram RVS e 2 sofreram intensificação da terapêutica hipoglicemiante. Dos restantes 17 doentes avaliados, constatámos que 2 diminuíram o número de fármacos realizado para controlo da DM2 e 15 mantiveram a terapêutica.

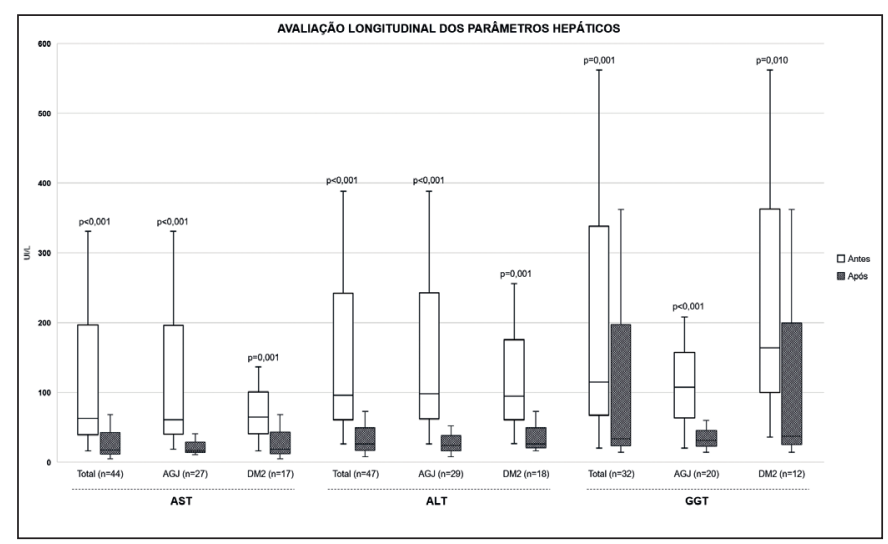

Figura 1. Avaliação longitudinal dos parâmetros hepáticos.
Assim, no total, 27 doentes tinham valores de glicemia em jejum e $11 \mathrm{HbA} 1 \mathrm{c}$ antes e após o tratamento. Verificámos que o valor da glicose em jejum melhorou significativamente após o tratamento. A HbA1c não foi significativamente diferente, no entanto a sua mediana após tratamento foi inferior à mediana pré-tratamento $(5,9 \%$ vs $6,1 \% ; p>0,05)$. Não houve diferenças significativas no peso antes e após o tratamento (Tabela 2).

\section{Determinar Fatores Preditivos de Melhoria do Metabolismo da Glicose}

Verificámos que a fibrose antes do tratamento se relacionou significativamente e de forma inversa com a variação da glicose em jejum, isto é, quanto menor a fibrose inicial, maior foi a variação da glicose com o tratamento. Não encontrámos correlações significativas da variação da glicose com outras variáveis (Tabela 3 ).

$\mathrm{Na}$ tentativa de determinar eventuais fatores associados à melhoria do metabolismo da glicose após cura do VHC, analisámos a variação do valor de glicose em jejum antes e após o tratamento antivírico. A mediana da variação da glicose em jejum global foi de $-12 \mathrm{mg} / \mathrm{dL}$, tendo sido significativamente mais elevada nos doentes com DM2 (-17,5 mg/dL; (P25 - 44.5; P75 -12) (mínimo - 74; máximo - 4)) do que nos doentes com AGJ (- $8 \mathrm{mg} / \mathrm{dL})(\mathrm{P} 25$-13; P75 -1) (mínimo - 39; máximo - 4) $(p=0,021)$.

Procedemos a uma análise de regressão longitudinal para avaliar eventuais fatores preditores de melhoria da glicemia em jejum apos o tratamento com AAD. Considerámos o outcome "variação

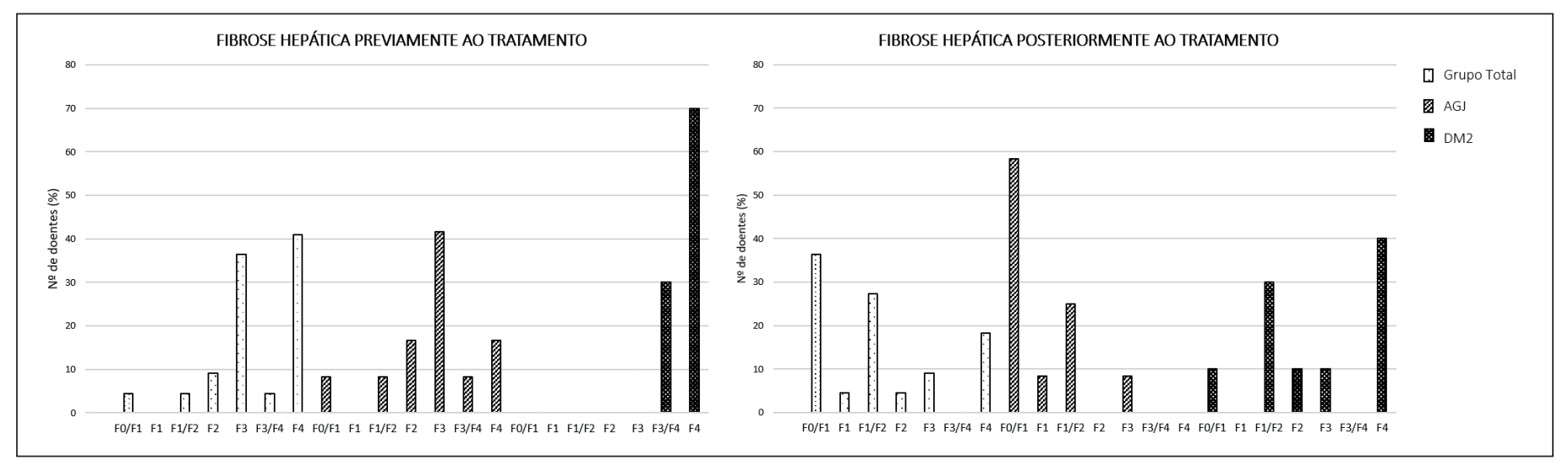

Figura 2. Avaliação longitudinal da fibrose hepática (grupo total: $p=0,001, \mathrm{AGJ}: p=0,005, \mathrm{DM} 2: p=0,027$ ) 
Tabela 3. Correlação entre a variação da glicose em jejum após o tratamento e características demográficas, relacionadas com a infeção por VHC, parâmetros hepáticos e metabólicos.

\begin{tabular}{lcc}
\hline & $\boldsymbol{r}$ & $\boldsymbol{p}$ \\
\hline Idade $(\mathbf{n}=\mathbf{2 7})$ & $-0,044$ & NS \\
\hline Peso prévio (n=22) & $-0,003$ & NS \\
\hline Tempo desde diagnóstico até tratamento $(\mathbf{n}=\mathbf{1 9})$ & $-0,259$ & NS \\
\hline Carga vírica prévia $(\mathbf{n}=\mathbf{2 7})$ & $-0,155$ & NS \\
\hline AST prévia $(\mathbf{n}=\mathbf{2 7})$ & $-0,255$ & NS \\
\hline ALT prévia $(\mathbf{n}=\mathbf{2 7})$ & $-0,077$ & NS \\
\hline G-GT prévia $(\mathbf{N}=\mathbf{2 4})$ & $-0,346$ & NS \\
\hline Fibrose prévia $(\mathbf{n}=\mathbf{2 2})$ & $-0,459$ & 0.032 \\
\hline$\Delta$ Peso $(\mathbf{n}=\mathbf{1 9})$ & $-0,027$ & NS \\
\hline$\Delta$ AST $(\mathbf{n}=\mathbf{2 6})$ & 0,282 & NS \\
\hline$\Delta$ ALT $(\mathbf{n}=\mathbf{2 7})$ & 0,025 & NS \\
\hline$\Delta \mathbf{G - G T}(\mathbf{N}=\mathbf{1 8})$ & 0,110 & NS \\
\hline
\end{tabular}

* Média \pm desvio padrão (mínimo-máximo) ** Mediana (percentis 25 - 75) (mínimo - máximo); AST: aspartato aminotranferase ALT: alanina aminotranferase GGT: gama glutamil transferase NS: não significativo percentual da glicemia em jejum $>7 \%$ ", uma vez que este valor correspondia à mediana da variação da glicose em jejum no grupo em que esta foi menor (doentes com AGJ: variação mediana da glicemia em jejum: $8 \mathrm{mg} / \mathrm{dL}$, correspondendo a uma variação percentual de 7\%). Construímos vários modelos de regressão logística univariada para analisar eventuais fatores preditores deste outcome. Considerámos potenciais preditores características demográficas, tempo de infeção pré-tratamento, carga vírica pré-tratamento, parâmetros hepáticos que sofreram melhoria significativa com o tratamento, assim como as suas respetivas variações (valor após tratamento - valor pré-tratamento) e parâmetros metabólicos considerados relevantes (glicose em jejum prévia, peso prévio e variação do peso). Nenhum destes parâmetros foi identificado como preditor do outcome definido (Tabela 4).

\section{Discussão}

A infeção por VHC é uma causa major de doença hepática crónica. ${ }^{4}$ Atualmente, com os novos esquemas terapêuticos compostos por $\mathrm{AAD}$, que apresentam um perfil de segurança favorável, tem-se verificado um aumento significativo na obtenção de resposta virológica sustentada, que se aproxima de $100 \% .{ }^{16}$ A cura da infeção associa-se a melhoria da necroinflamação hepática e fibrose, e ainda

Tabela 4. Modelos de regressão logística univariada tendo como outcome a redução de, pelo menos, 7\% no valor da glicemia em jejum após o tratamento antivírico.

\begin{tabular}{|c|c|c|c|c|c|}
\hline & \multicolumn{2}{|c|}{ Redução da glicemia em jejum $\geq 7 \%$} & \multirow[b]{2}{*}{$p$} & \multirow[b]{2}{*}{ OR } & \multirow[b]{2}{*}{$95 \%$ IC } \\
\hline & Não & Sim & & & \\
\hline \multirow{3}{*}{$\begin{array}{l}\text { Sexo } \\
\text { Masculino } \\
\text { Feminino }\end{array}$} & $\mathrm{n}=10$ & $\mathrm{n}=17$ & \multirow{3}{*}{ NS } & \multirow{3}{*}{0,533} & \multirow{3}{*}{$0,063-4,531$} \\
\hline & $8(80 \%)$ & $15(88,2 \%)$ & & & \\
\hline & $2(20 \%)$ & $2(11,8 \%)$ & & & \\
\hline \multirow{2}{*}{ Idade* } & $\mathrm{n}=10$ & $\mathrm{n}=17$ & \multirow{2}{*}{ NS } & \multirow{2}{*}{1,014} & \multirow{2}{*}{$0,948-1,085$} \\
\hline & $55,6 \pm 12,1(32-75)$ & $57,53 \pm 12,2(40-82)$ & & & \\
\hline \multirow{2}{*}{ Tempo desde diagnóstico até tratamento** } & $\mathrm{n}=8$ & $\mathrm{n}=11$ & \multirow{2}{*}{ NS } & \multirow{2}{*}{1,067} & \multirow{2}{*}{$0,963-1,183$} \\
\hline & $2,5(1-20,8)(1-21)$ & $12(5-20)(3-32)$ & & & \\
\hline \multirow{3}{*}{ Carga vírica antes** } & $\mathrm{n}=10$ & $\mathrm{n}=17$ & \multirow{3}{*}{ NS } & \multirow{3}{*}{1,000} & \multirow{3}{*}{$1,000-1,000$} \\
\hline & $3138821(111229,3-5738802,8)$ & $2169868(1270739,5-4136787)$ & & & \\
\hline & $(66953-9270580)$ & $(128-13487898)$ & & & \\
\hline \multirow{2}{*}{$\operatorname{AST}(\mathrm{UI} / \mathrm{L})^{* *}$} & $\mathrm{n}=10$ & $\mathrm{n}=17$ & \multirow{2}{*}{ NS } & \multirow{2}{*}{1,033} & \multirow{2}{*}{$0,996-1,073$} \\
\hline & $43(22,3-62,5)(19-72)$ & $67(39,5-87)(16-320)$ & & & \\
\hline \multirow{2}{*}{$\Delta \mathrm{AST}^{* *}$} & $\mathrm{n}=10$ & $\mathrm{n}=16$ & \multirow{2}{*}{ NS } & \multirow{2}{*}{0,976} & \multirow{2}{*}{$0,945-1,008$} \\
\hline & $-21,5(-40,3 ;-8,3)(-56 ;-5)$ & $-48(-71,3 ;-23,5)(-283 ; 25)$ & & & \\
\hline $\operatorname{ALT}(\mathrm{UI} / \mathrm{L}) * *$ & $\mathrm{n}=10$ & $\mathrm{n}=17$ & NS & & \\
\hline $\operatorname{ALI}(\mathrm{UI} / \mathrm{L})^{*}$ & $77,5(48,3-112,8)(27-133)$ & $94(51-178)(27-388)$ & NS & 1,011 & $0,995-1,028$ \\
\hline$\triangle \mathrm{ALT} * *$ & $\mathrm{n}=10$ & $\mathrm{n}=17$ & NS & 0.992 & \\
\hline$\triangle \mathrm{ALI}$ & $-54,5(-84,5 ;-25)(-109 ;-15)$ & $-67(-145 ;-23)(-358 ; 25)$ & NS & 0,992 & $0,9 / 8-1,006$ \\
\hline GGT $(\mathrm{UI} / \mathrm{L}) * *$ & $\mathrm{n}=9$ & $\mathrm{n}=15$ & NS & 1003 & $0.995-1012$ \\
\hline GU1 (UI/L) & $63(45,3-137)(20-423)$ & $115(65-208)(34-507)$ & NS & 1,003 & $0,993-1,012$ \\
\hline$\Delta \mathrm{GGT}^{* *}$ & $\mathrm{n}=9$ & $\mathrm{n}=9$ & NS & 1 & $0.991-1009$ \\
\hline & $-42(-108 ;-16)(-399 ;-6)$ & $-79(-166,5 ;-40.5)(-257 ; 110)$ & & & \\
\hline Fibrose hepática pré- tratamento & $\mathrm{n}=10$ & $\mathrm{n}=17$ & & & \\
\hline $\mathrm{F} 0 / \mathrm{F} 1$ & $2(22,2 \%)$ & $2(15,4 \%)$ & & & \\
\hline $\mathrm{F} 1 / \mathrm{F} 2$ & $2(22,2 \%)$ & - & NS & 1301 & $0.928-1823$ \\
\hline $\mathrm{F} 2$ & $1(11,1 \%)$ & $1(7,7 \%)$ & NS & 1,301 & $0,928-1,823$ \\
\hline F3 & $2(22,2 \%)$ & $2(15,4 \%)$ & & & \\
\hline F4 & $2(22,2 \%)$ & $8(61,5 \%)$ & & & \\
\hline Melhoria da fibrose hepática & $\mathrm{n}=5$ & $\mathrm{n}=9$ & NS & & \\
\hline Sim & $4(80 \%)$ & $6(66,7 \%)$ & NS & 0,5 & $0,037-6,683$ \\
\hline Glicose em ieium antes $(\mathrm{mo} / \mathrm{dL}) * *$ & $\mathrm{n}=10$ & $\mathrm{n}=17$ & NS & 1013 & $0977-1049$ \\
\hline Glicose em jejum antes (mg/aL) & $110,5(103,5-118)(102-174)$ & $111(104,5-132)(101-197)$ & NS & 1,013 & $0,911-1,049$ \\
\hline Peso antes $(\mathrm{kg}) * *$ & $\mathrm{n}=9$ & $\mathrm{n}=13$ & NS & 1007 & $0.961-1056$ \\
\hline Peso antes (Kg) & $72(66-92)(62-98)$ & $74,5(-61,2-98,5)(50-128)$ & NS & $1,00 /$ & $0,901-1,050$ \\
\hline Variação do peso $(\mathrm{kg})^{* *}$ & $\begin{array}{c}\mathrm{n}=8 \\
-0.7(-6,5 ; 2)(-10 ; 2,3)\end{array}$ & $\begin{array}{c}\mathrm{n}=11 \\
-1(-3,5 ; 3,7)(-20 ; 5,5)\end{array}$ & NS & 1,018 & $0,872-1,188$ \\
\hline
\end{tabular}

\footnotetext{
* Média \pm desvio padrão (mínimo-máximo) ** Mediana (percentis 25 - 75) (mínimo - máximo); AST: aspartato aminotranferase ALT: alanina aminotranferase GGT: gama glutamil transferase NS: não significativo
} 
reversão da maioria das manifestações extra-hepáticas associadas ao VHC. ${ }^{4}$ Assim, este estudo pretendeu avaliar o impacto dos AAD na eventual melhoria do metabolismo da glicose.

No presente estudo foram analisados 138 doentes sujeitos a erradicação vírica com AAD no Hospital de Braga entre 2015 e 2019 , sendo que $70 \%$ destes doentes nunca tinham sido tratados para a infeção por HCV. Trinta e um por cento apresentavam cirrose e cerca de metade tinham excesso de peso ou obesidade. Trinta e nove por cento tinham alterações do metabolismo da glicose, tendo $22,5 \%$ AGJ e $16,6 \%$ DM2, valores consistentes com os dados reportados na literatura. ${ }^{10,17}$

Os doentes com alterações do metabolismo da glicose tinham maior grau de fibrose e uma prevalência superior de cirrose comparativamente aos doentes sem alterações do metabolismo da glicose (42\% vs 24\%). Estes doentes tinham ainda níveis mais elevados de GGT e maior compromisso da função hepática. A associação entre fibrose hepática e insulinorresistência/hiperglicemia parece ser bidirecional, já que a insulinorresistência parece ser fator preditor independente de fibrose hepática avançada ${ }^{18} \mathrm{e}$ a fibrose hepática severa e cirrose, por sua vez, foram associadas ao desenvolvimento de DM2. ${ }^{11,13}$ Assim se poderá explicar o pior perfil hepático e a maior prevalência de fibrose avançada e cirrose nos doentes com DM2 relativamente aos doentes com AGJ, que se encontrou neste estudo.

Com a eliminação vírica, verificada na quase totalidade dos doentes, houve diminuição da fibrose e do nível das transaminases e da GGT, o que traduz uma melhoria precoce da doença hepática pré-existente, tal como reportado na literatura. ${ }^{4,15,19}$ A cura da infeção associou-se, também, a uma melhoria do status metabólico, com redução significativa do valor da glicose em jejum. A melhoria da glicose em jejum ocorreu independentemente do peso, que não sofreu alterações significativas. Estes resultados estão descritos, principalmente em populações de doentes com DM2. ${ }^{15,17,20-22}$ Também se verificou uma diminuição do valor de HbA1c nos doentes com DM2, ainda que sem significado estatístico, porventura pelo reduzido número de doentes e/ou pelo facto de a maioria dos doentes diabéticos já ter valores de $\mathrm{HbA} 1 \mathrm{c}$ prévios no objetivo terapêutico. Há estudos que reportam melhoria dos valores de HbAlc após o tratamento, ${ }^{15,20,23}$ mas esta conclusão ainda não é consensual. ${ }^{24,25}$

A correlação inversa encontrada entre o grau de fibrose hepática antes do tratamento e variação de glicose após o tratamento parece apontar para uma maior capacidade de reversão da alteração do metabolismo da glicose nos doentes com menor grau de atingimento hepático, o que está de acordo com outro estudo que verificou associação entre a severidade da doença hepática segundo a classificação de Child Pugh e melhoria do controlo glicémico. ${ }^{26}$

No entanto, o facto de não se ter encontrado correlação entre a variação da glicose e outros parâmetros hepáticos pré-tratamento e a sua variação com o tratamento, pode apontar para que não seja apenas pela melhoria do status hepático que se verifica a melhoria metabólica. Não se pode excluir a existência de outros mecanismos que expliquem a relação entre a infeção por VHC e as alterações do metabolismo da glicose. ${ }^{10-13}$ Um estudo recente corrobora esta conclusão, pois demonstra uma melhoria significativa do controlo glicémico em pacientes com hepatite $\mathrm{C}$ crónica, mesmo quando se encontram em estadio de cirrose hepática. ${ }^{27}$

Não foi possível identificar preditores de atingimento do outcome definido por diminuição da glicemia em jejum superior ou igual a 7\%. Tal pode ter ocorrido pelo reduzido número de doentes ou porque o outcome definido não é o que melhor reflete a associação entre as variáveis estudadas e a melhoria da glicose em jejum. A possibilidade de, em estudos futuros, incluir um maior número de doentes, poderá permitir a utilização de modelos de regressão linear, usando como outcome a variação absoluta ou percentual da glicose sem definir cut-offs, o que pode permitir encontrar eventuais associações.

\section{Limitações do Estudo}

As conclusões deste estudo são limitadas por vários fatores, nomeadamente, e com maior peso, o reduzido tamanho amostral, juntamente com o seu carácter retrospetivo. Para além disso, há poucos dados relativos aos valores da $\mathrm{HbAlc}$, o que poderia contribuir para uma melhor avaliação da evolução do controlo glicémico. A utilização de um valor de glicemia em jejum pré-tratamento e um valor de glicemia em jejum após o tratamento é também uma limitação deste trabalho, pois não permitem averiguar a sustentabilidade da resposta metabólica à eliminação do vírus. Por último, alterações do estilo de vida, como melhoria da dieta ou prática de exercício físico, não foram avaliadas, apesar de poderem ter um papel importante no controlo glicémico.

Assim, estudos prospetivos devem ser desenvolvidos no sentido de averiguarem a sustentabilidade desta resposta após cura da infeção por VHC e identificar possíveis preditores do alcance de uma melhoria no controlo metabólico destes doentes. A criação de protocolos de avaliação metabólica prospetiva nestes doentes permitirá analisar com maior detalhe a melhoria do controlo glicémico que parece acompanhar a cura da infeção por VHC e esclarecer melhor o mecanismo que lhe está subjacente.

\section{Conclusão}

Nesta população de doentes com infeção por HCV e alterações do metabolismo da glicose, verificou-se uma melhoria do controlo glicémico após a eliminação da infeção por $\mathrm{HCV}$, quer em doentes com DM2, quer em doentes com alteração da glicemia em jejum. Os parâmetros hepáticos analíticos normalizaram e ocorreu regressão da fibrose, o que pode ter contribuído para a melhoria da disfunção metabólica.

É crescente a evidência de que curar a infeção por VHC com AAD tem impacto na melhoria do controlo glicémico em doentes com DM2 e pré-DM2. São, no entanto, necessários mais estudos para esclarecer com maior precisão os mecanismos subjacentes a esta melhoria. Por último, a eliminação do vírus, se feita precocemente, poder eventualmente evitar aparecimento de novos casos de pré-DM2 e DM2 associados a esta infeção.

\section{Responsabilidades Éticas}

Conflitos de Interesse: Os autores declaram a inexistência de conflitos de interesse na realização do presente trabalho.

Fontes de Financiamento: Não existiram fontes externas de financiamento para a realização deste artigo.

Confidencialidade dos Dados: Os autores declaram ter seguido os protocolos da sua instituição acerca da publicação dos dados de doentes.

Proteção de Pessoas e Animais: Os autores declaram que os procedimentos seguidos estavam de acordo com os regulamentos estabelecidos pelos responsáveis da Comissão de Investigação Clínica e Ética e de acordo com a Declaração de Helsínquia revista em 2013 e da Associação Médica Mundial.

Proveniência e Revisão por Pares: Não comissionado; revisão externa por pares. 


\section{Ethical Disclosures}

Conflicts of Interest: The authors have no conflicts of interest to declare.

Financing Support: This work has not received any contribution, grant or scholarship

Confidentiality of Data: The authors declare that they have followed the protocols of their work center on the publication of data from patients.

Protection of Human and Animal Subjects: The authors declare that the procedures followed were in accordance with the regulations of the relevant clinical research ethics committee and with those of the Code of Ethics of the World Medical Association (Declaration of Helsinki as revised in 2013).

Provenance and Peer Review: Not commissioned; externally peer reviewed.

\section{References / Referências}

1. Who.int. Hepatitis C. [consultado em 2019 agosto 26] Disponível em: https://www.who.int/news-room/factsheets/detail/hepatitis-c

2. Spengler U. Direct antiviral agents (DAAs) - A new age in the treatment of hepatitis C virus infection. Pharmacol Ther. 2018;183:118-126. doi: 10.1016/j.pharmthera.2017.10.009.

3. World Health Organization. Guidelines for the screening, care and treatment of persons with chronic hepatitis c infection. Geneva: WHO;2016.

4. European Association for the Study of the Liver. EASL Recommendations on Treatment of Hepatitis C 2018. J Hepatol. 2018;69:461-511. doi: org/10.1016/j. jhep.2018.03.026

5. American Diabetes Association. Diagnosis and Classification of Diabetes Mellitus. Diabetes Care. 2003; 27: S5-S10. doi: 10.2337/diacare.27.2007. s5

6. American Diabetes Association. Classification and diagnosis of diabetes: Standards of Medical Care in Diabetes 2019. Diabetes Care. 2019;42:S13S28. doi.org/10.2337/dc19-S002

7. UpToDate. Insulin resistance: Definition and clinical spectrum [consultado em 2019 agosto 26] Disponível em: https://www.uptodate.com/contents/ insulin-resistance-definition-and-clinicalspectrum\#H2

8. Antuna-Puente B, Disse E, Rabasa-Lhoret R, Laville M, Capeau J, Bastard J. How can we measure insulin sensitivity/resistance? Diabetes Metab. 2011;37:179-88. doi: 10.1016/j.diabet.2011.01.002

9. Desbois AC, Cacoub P. Diabetes mellitus, insulin resistance and hepatitis $\mathrm{C}$ virus infection: A contemporary review. World J Gastroenterol. 2017;23:1697-711. doi: 10.3748/wjg.v23.i9.1697.

10. Gastaldi G, Goossens N, Clément S, Negro F. Current level of evidence on causal association between hepatitis $\mathrm{C}$ virus and type 2 diabetes: A review. J Adv Res. 2017;8:149-59. doi: 10.1016/j.jare.2016.11.003.

11. García-Compeán D, González-González JA, Lavalle-González FJ, González-Moreno EI, Villarreal-Pérez JZ, Maldonado-Garza HJ. Current Concepts in Diabetes Mellitus and Chronic Liver Disease: Clinical Outcomes, Hepatitis C Virus Association, and Therapy. Dig Dis Sci. 2016;61):371-80. doi: 10.1007/s10620-015-3907-2.

12. Serfaty L. Metabolic Manifestations of Hepatitis C Virus: Diabetes Mellitus, Dyslipidemia. Clin Liver Dis. 2017;21:475-86. doi: 10.1016/j. cld.2017.03.004.
13. Hammerstad SS, Grock SF, Lee HJ, Hasham A, Sundaram N, Tomer Y Diabetes and Hepatitis C: A Two-Way Association. Front Endocrinol. 2015;6:134. doi: 10.3389/fendo.2015.00134.

14. Vanni E, Bugianesi E, Saracco G. Treatment of type 2 diabetes mellitus by viral eradication in chronic hepatitis C: Myth or reality? Dig Liver Dis 2016;48:105-11. doi: 10.1016/j.dld.2015.10.016.

15. Attia M, Hashim A, Kandeel H, Hendy O, El-Mola K, El-Raey F. Effect of new direct-acting antiviral drugs on insulin resistance and glycemic control after treatment of chronic hepatitis $\mathrm{C}$ virus infection in type 2 diabetic patients. Al-Azhar Assiut Med J. 2017;15:187. doi: 10.4103/ AZMJ.AZMJ_7_18

16. Asselah T, Marcellin P, Schinazi RF. Treatment of hepatitis C virus infection with direct-acting antiviral agents: $100 \%$ cure? Liver Int. 2018;38 Suppl 1:7-13. doi: 10.1111/liv.13673.

17. Weidner P, Boettche D, Zimmerer T, Burgermeister E, Teufel A, Ebert $\mathrm{MP}$, et al. Impact of direct acting antiviral (DAA) treatment on glucose metabolism and reduction of pre-diabetes in patients with chronic hepatitis C. J Gastrointestin Liver Dis. 2018;27:281-9. doi: 10.15403/ jgld.2014.1121.273.daa.

18. Sert A, Pirgon O, Aypar E, Yılmaz H, Dündar B. Relationship between aspartate aminotransferase-to-platelet ratio index and carotid intimamedia thickness in obese adolescents with non-alcoholic fatty liver disease. J Clin Res Pediatr Endocrinol. 2013;5:182-8. doi: 10.4274/ Jcrpe. 891.

19. Persico M, Rosato V, Aglitti A, Precone D, Corrado M, De Luna A, et al Sustained virological response by direct antiviral agents in HCV leads to an early and significant improvement of liver fibrosis. Antivir Ther. 2018;23:129-38. doi: 10.3851/IMP3186.

20. Ciancio A, Bosio R, Bo S, Pellegrini M, Sacco M, Vogliotti E, et al. Significant improvement of glycemic control in diabetic patients with $\mathrm{HCV}$ infection responding to direct-acting antiviral agents. J Med Virol. 2018;90:320-27. doi: 10.1002/jmv.24954.

21. Pavone P, Tieghi T, d'Ettorre G, Lichtner M, Marocco R, Mezzaroma I, et al. Rapid decline of fasting glucose in HCV diabetic patients treated with direct-acting antiviral agents. Clin Microbiol Infect. 2016;22:462.e1-3. doi: $10.1016 / \mathrm{j} . \mathrm{cmi} .2015 .12 .030$.

22. Drazilova S, Janicko M, Skladany L, Kristian P, Oltman M, Szantova M, et al. Glucose Metabolism Changes in Patients with Chronic Hepatitis C Treated with Direct Acting Antivirals. Can J Gastroenterol Hepatol. 2018;2018:6095097. doi: 10.1155/2018/6095097.

23. Hum J, Jou JH, Green PK, Berry K, Lundblad J, Hettinger BD, et al. Improvement in Glycemic Control of Type 2 Diabetes After Successful Treatment of Hepatitis C Virus. Diabetes Care. 2017;40:1173-80. doi: $10.2337 / \mathrm{dc} 17-0485$.

24. Chaudhury CS, Sheehan J, Chairez C, Akoth E, Gross C, Silk R, Kattakuzhy S, Rosenthal E, Kottilil S, Masur H, Hadigan C. No Improvement in Hemoglobin A1c Following Hepatitis C Viral Clearance in Patients With and Without HIV. J Infect Dis. 2017;217:47-50. doi: 10.1093/infdis/jix517.

25. Stine JG, Wynter JA, Niccum B, Kelly V, Caldwell SH, Shah NL. Effect of Treatment with Direct Acting Antiviral on Glycemic Control in Patients with Diabetes Mellitus and Chronic Hepatitis C. Ann Hepatol. 2017;16:215-20. doi: 10.5604/16652681.1231581.

26. Dawood AA, Nooh MZ, Elgamal AA. Factors Associated with Improved Glycemic Control by Direct-Acting Antiviral Agent Treatment in Egyptian Type 2 Diabetes Mellitus Patients with Chronic Hepatitis C Genotype 4. Diabetes Metab J. 2017;41:316-21. doi: 10.4093/dmj.2017.41.4.316.

27. Lanini S, Bartolini B, Taibi C, Agresta A, Garbuglia AR, Montaldo C, et al. Early improvement of glycaemic control after virus clearance in patients with chronic hepatitis $\mathrm{C}$ and severe liver fibrosis: a cohort study. New Microbiol. 2019;42:139-44. 